DOI: https://doi.org/10.36548/jucct.2020.1.006

\title{
A Secure Protocol for Smart Meters using IoT Enabled Distribution Networks and Blockchain Security Mechanism
}

\author{
Madhura S \\ Assistant Professor, \\ Department of Electronics and Communication Engineering, \\ RV Institute of Technology and Management, \\ Bangalore, India \\ Email: madhuras.rvitm@rvei.edu.in \\ Thilak Raj L \\ Manager Expert, \\ CGI Information Systems and Management Consultants Private Ltd \\ Email: thilaakraj@gmail.com
}

\begin{abstract}
Smart Cities, Homes and Communities are the spaces where IoT sensors are used to the fullest in the Utilities sector. Advanced Metering Infrastructure (AMI) providing the peer-to-peer communication between metering equipment that reads, calculate and provides the information related to distribution and measure the consumption. This paper outlines the network monitoring and management architecture that can be used in Smart Cities, homes and communities using the integrated IoT and Blockchain technology. Blockchain technology provides trustworthy resource monitoring of utilities for all members of the smart community that in-turn benefit the members of smart communities with enhanced monitoring and optimize the consumption of resources in protected and transparent way.
\end{abstract}

Key words: Smart Meter, Block chain technology, IoT, Energy consumption, Time-of-Use [ToU]

\section{Introduction}

A smart metermakes smart city, better utilization of the data from these meters with better security makes the system more robust and flexible. Of course with the increased smart meter installation energy wastage has cropped down [1],[2]. This can be achieved through integration of IoT devices with meter abled with peer-to-peer secure and safe transaction tracking methods.Generally, smart meters assist in developing a customized retailing procedure containing High Quality Information [HQI] measured quantitatively. These data ascertain the pattern or the rhythm in electricity consumption by the customers to arrive at a logical optimization of energy conservation and utilization sequence. 
Journal of Ubiquitous Computing and Communication Technologies (UCCT) (2020)

Vol.02/ No. 01 Pages: 48-58

https://www.irojournals.com/jucct/

DOI: https://doi.org/10.36548/jucct.2020.1.006

A consumer requires continuous reading, recording and interpretation of the smart meter data from the utilities like gas, electric and/or water on a daily basis. This would influence a lot of other factors like power distribution analysis, retailer level utilization, consumer billing, peak hour monitoring, time-controlled demand monitoring, service and outage monitoring. All the above factors can be monitored using sensor based intelligent real-time communication system using Internet of things (IoT). They form the basic building blocks for data collection, monitoring and controlling of devices remotely [3].

Vividconsumer satisfaction [4] can be targeted through smart and safe metering technique that increases the profit margin of the retailers with high permeability in market stability. In the current scenario there is a huge demand for research and innovation in merging of metered data with the authentication from a secured platform, which can be provided by a most rated and retailer trusty technology called Blockchain. Blockchain technology is one of the fastest growing technique which is attracting most of the researchers and of course huge funding is happening in this area of research. Blockchains are distributed (i.e., without a central repository), tamper resistant, sharing platforms that allows multiple authoritative domains, who do not trust each other, to work together in application development process or business development process. Blockchain can be thought of as a distributed database which accommodates and thus helps in cooperation between multiple authoritative domains providing strong consistency support. Many of the developed nations such as Australia, USA and UK have retail markets where purchase of energy not only happen through spot markets nut also by bilateral contracts since marketers might not have generating stations of their own.Electricity Generation Supplier in UK is the Big Six Energy suppliers who sell the power directly to the customers [5] who uses over-the-counter trading method.In USA Electric Energy suppliers are mainly FirstEnergy and American Electric Power Energy who rely on self-produced generation sold by investor Owned Utilities[6].These suppliers are self-operated with automated bill generation for customers. But there is a trade-off between the generation asset and the suppliers profit as the utilization relays on the load profiles. The greater challenge is consideration of utilities infrastructure for every smart meter which is influenced by various factors like: region bias for new technology to be installed in smart meters, infrastructure short fall in communication network, and dependency on external devices for communication, necessary skilled labours for meter monitoring and data leakage and theft.

In our paper we have proposed blockchain technique to address some of the issues stated above. Few key features of the blockchain like disabled editing options, multiple node access and maintaining history of every change makes its unique in comparison with generic database. Added new feature of this technique is the automated authentication by smart contacts or chaincode which follow predefined protocol for Internet of Things [IoT] platform. We propose a unique integrated IoT architecture for monitoring, recording and securing the data obtained from smart meters of gas, water and electricity usage which provides accurate and reliable billing system beneficial for both customers and suppliers. 
The current session provided the details of the state-of-art technology used in our proposed architecture. The structure of the papers is as follows: in section 2, describes the proposed design and key features of the smart meter. Section 3 contains the communication protocol with IoT integration. Section 4 compares the proposed technique with the existing methodology and analysis the results. Section 5 describes the integration of IoT data with the blockchain and communication between the utilities and customers. Section 6 briefs the advantages and improvements made towards better energy utilization of the proposed architecture. Finally, in section 7 conclusion and future work are explained.

\section{Design specification of smart meters}

Utilities get the required information regarding the energy usage from gas, water and electricity by the smart meters. Along with these the peer-to-peer communication protocol provides a detail description of the realtime consumption by the consumer to the utilities and also customer can be aware of the generation assets. Smart meters not only benefit the customers and retailers, the information gathered helps in improvisation of the communication network, understand the Time-Of-Use [TOU] range, customization of the security keys and real-time data exchange helps in energy saving. With refence to the above features of the smart meters it is necessary to understand the functional aspects of these meters. Some of the key challenges that needs to be addressed include:

1. Battery life of the smart meters needs a constant monitoring and replacement [7]

2. Data management servers to handle the real-time data flow which includes quantitative readings from demand network and frequency management[8]

3. Geographical limitations of the smart meters: it might take few hours to couple of days for the recorded data to reach the main utility server posing a great challenge for real-time processing.

4. Blockchain framework is introduced for authentication and validation of the data, there can be occurrence of human errors during configuration which needs to be addressed.

5. Any changes like addition or deletion of the data or block requires a constant monitoring of priority level affecting the device configuration.

6. Recording of all the changes maintained in blockchain requires evaluation by audit and security teams. 
Journal of Ubiquitous Computing and Communication Technologies (UCCT) (2020)

Vol.02/ No. 01 Pages: 48-58

https://www.irojournals.com/jucct/

DOI: https://doi.org/10.36548/jucct.2020.1.006

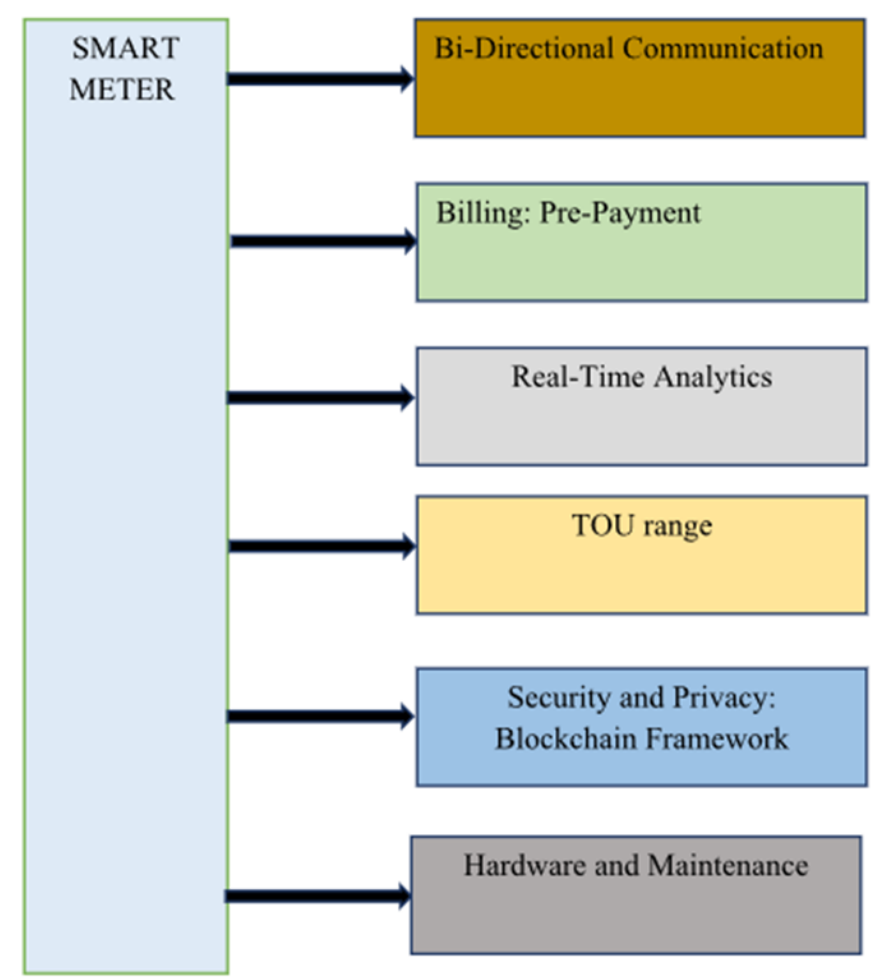

Fig 1: Features of Smart meter

Fig 1 shows the features of the smart meter as a basic requirement for energy saving and security maintenance system. The data communication is bidirectional with a facility to make pre-payments. The large data movements open up an arena of frequency reading and monitoring technique understanding for real-time implementation. The study of TOU range provides marginal cost difference between generation cost and utilization at different periods in wholesale market.It provides a trade-off for improvisation of energy consumption behaviour of customers.

\section{Communication and Architecture Protocol}

The architectural protocol has been broken into two layers for better classification and understanding of the interconnections. These two layers are further fit to three different levels: Power transmission level, power distribution level and customer level as shown in fig 2---. The first layer is the AMI power system which includes generating stations and utilities, substation, smart meters, hardware, sensors, communications and displays. There is always a two-way communication established between the generation utilities and the 
end user where the commands can be sent from utilities to customer smart meters with real-time data and receive the changes made from the customers. The power distribution level is responsible for distribution of information to and from the utilities and the smart meters, retailers, suppliers and facilities. Layer 2 consists of AMI communication system which has smart meters, all the information clustered as huge data driven through specific protocol, data management servers, metering servers, control system, billing system and the management system.

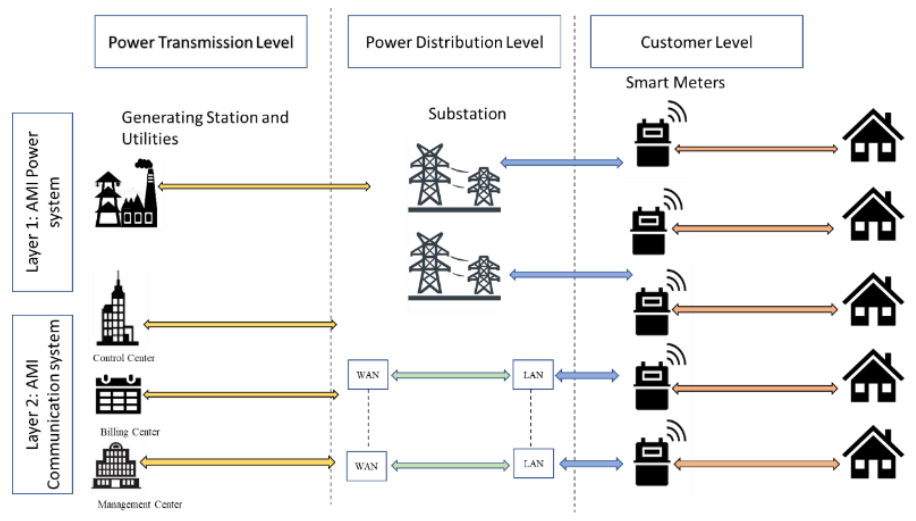

Fig 2: Proposed communication and power distribution architecture

The communication system has different levels of data exchange from Local area to house area networks with more than one decision making environment. It has to be noted that each of these networks has varied communication techniques. The smart meters already installed are either confined to particular location or limited communication being established. It is a great challenge to select a smart meter that addresses all the criteria in real-time. Absence of standard communication protocol restrains data exchanges between smart meter of different manufactures. However, with the availability of open access communication protocol by OMS-group [9], the communication system has become more robust and flexible, independent of manufacturer. The proposed architecture suggests various ways of data exchange compatible with standard communication protocol. The communication occurs between the utility and the customers either through the local or remote networks via internet. There is flexibility in type of hardware deployed: it can be wireless, wired, wireless fixed network or mix of any of the above. The type of technology and communication protocol to be used depends on various factors like utility acceptability, type of information extracted from the smart meters, billing and control system procedures, economical andcommercial aspects. 
Journal of Ubiquitous Computing and Communication Technologies (UCCT) (2020)

Vol.02/ No. 01 Pages: 48-58

https://www.irojournals.com/jucct/

DOI: https://doi.org/10.36548/jucct.2020.1.006

\section{Design of blockchain for network monitoring and authentication}

There are numerous smart meters available in market with several favourable features but providing security possess a biggest challenge in communication technology. Due to lack of computational capabilities of smart meters, cyber theft and misleading of data is increasing among the deployed meters.

Some of the cyber threats can be due to following reasons:

1. Tampering of data recordings for altering consumption details.

2. Destabilization of the generation assets by continuous altering commercial transactions for fraudulent purpose.

3. Unauthorised extraction of customer consumption pattern which greatly affects the privacy.

4. Access to smart meters wirelessly without authentication.

Blockchain technology is the most accepted and researched technology in the current tech industry. Bitcoin Cryptocurrency was the first to use blockchain as public ledger [10].Blockchain has two main characteristics: decentralization and democratic data control. The above features of the blockchain ensures that the data cannot be tempered and the history about the changes made to the configuration will be recorded and monitored. The blockchain used in bitcoin was public and permission less with limited flexibility[11]. This can be overcome by making the system private, and deploy a different strategy to get user control and execution. In this paper we propose a novel Blockchain technology that can be integrated with the IoT devices to record and monitor the smart meters. The complete design of the architecture is shown in Fig 3. There would be digital certificates for network authentication from authorized personal with access to modify the device configurations recorded in the blockchain.In [12] a blockchain based framework has been defined to provide authentication certificates which has been implemented in our paper rather than the Public Key Infrastructure [PKI].Verification of syntax is introduced to reduce the probability of human error in configuration maintained in the blockchain. For the identification of the authentic new configuration, an administration certificate is introduced. Any introduction of new block into the blockchain is complete, a complete transaction details are reported to blockchain network. The priority event is triggered to check whether modification in configuration is needed depending on the device ID. Then depending on the priority, the new encrypted configuration is downloaded from the block. The decryption of the configuration happens with the help of private key and the changes are reflected in the blockchain. Blockchain holds the lookup table containing all the details pertaining to the modification made to the blocks which can be reviewed for security and maintenance purpose. 


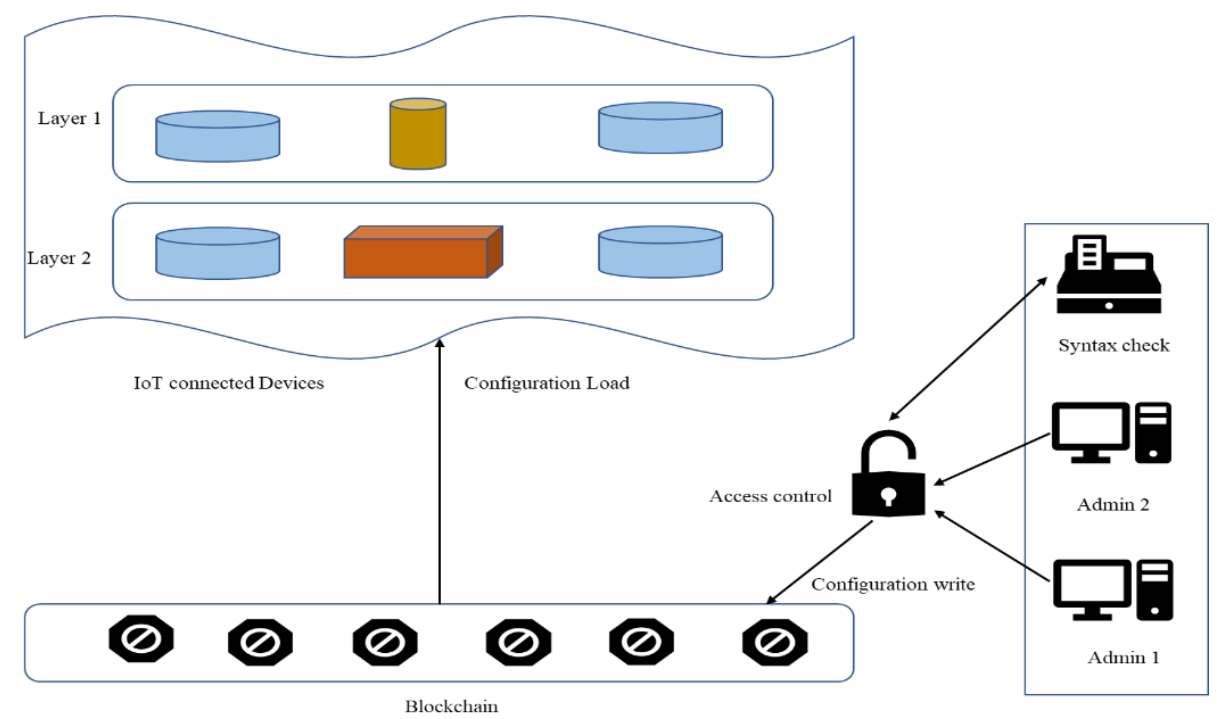

Fig 3: Blockchain authentication and control access architecture

\section{Results and Discussion}

The results from the experiment can be classified into two parts: firstly, understanding of the user consumption and TOU from the smart meter. Secondly analysis of the data and categorization of which can strategically requiresupdating of configuration. Smart meter data will be used to create user consumption frequency. Based on the patterns obtained there can be studies made on the gas, electricity and water management.Demand side analysis can be easy made with the data collected and energy conservation techniques from utilities can be better adapted. A data set comprising of smart electricity consumption readings and gas consumption of 5567 houses from London from a period of one-year 2018 were taken for analysis. The patterns can give information regarding number of devices used, duration of usage on hourly basis, compare the pricing during the utilization and create user profiles. Depending on the utilization the generation section can maximize the production of power to overcome the demand during peak hours. Fig 4shows the consumption pattern of electricity and gas from the real time data.In Fig 4(a) it can be observed that the electricity consumption is too high at 7 am that it doesn't last for more than few minutes, this indicates that its not the energy consumption data but some spike in the meter which needs to be analysed and monitored. In fig 4(b) the gas consumption for the entire year can be seen and it can be noticed that there is abrupt meter reading during February and December which is gain a spike. This can be analysed to find if there were any leak like the cistern leak. From this information the generation utilities will be able to detect the faults and also can request the customer to provide access to monitor and intimate the high consumption and demand statistics on the daily basis. 
Journal of Ubiquitous Computing and Communication Technologies (UCCT) (2020)

Vol.02/ No. 01 Pages: 48-58

https://www.irojournals.com/jucct/

DOI: https://doi.org/10.36548/jucct.2020.1.006

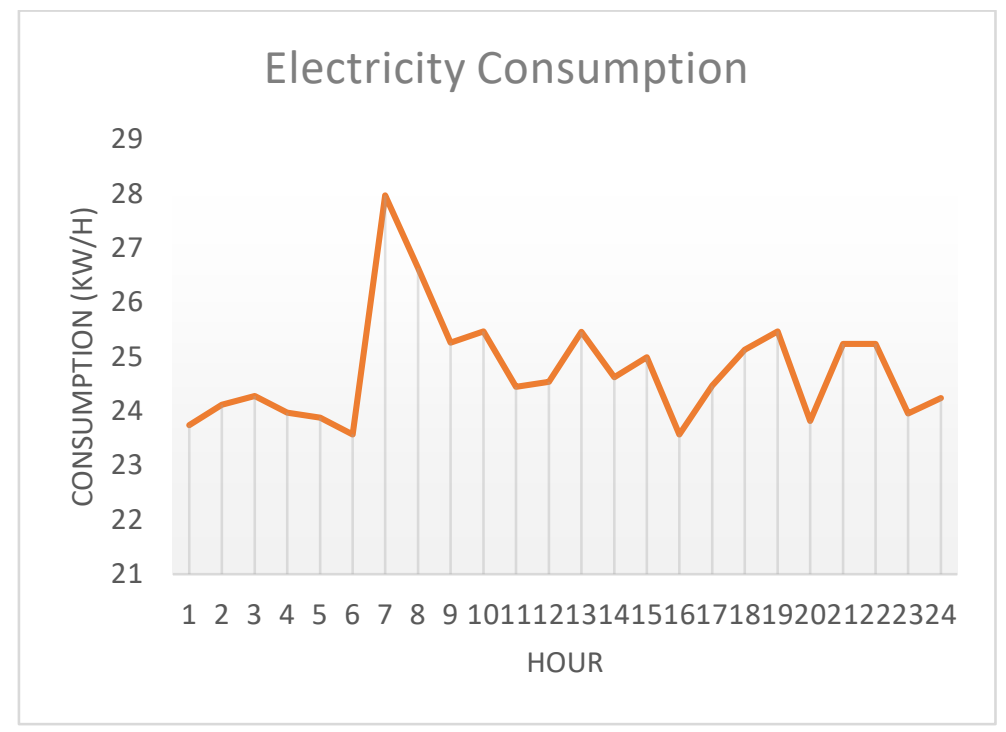

(a)

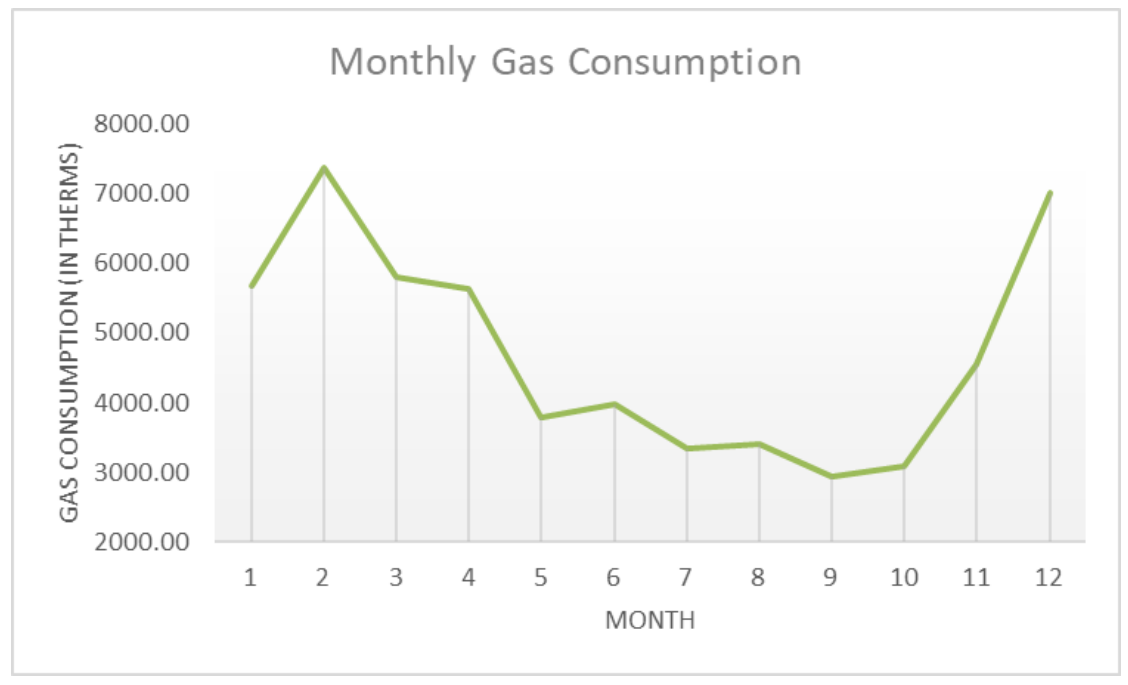

(b)

Fig 4: Electricity and Gas consumption pattern

For the device authentication and access control, the configuration can be implemented in Cisco environment. The procedure includes firstly detection of the device requesting for the data loading, secondly monitoring the time required for making the necessary upgradation in the block.Scripting

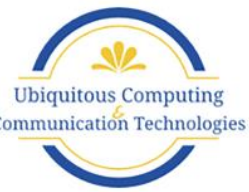


language like TCL will be used to upload and download the configuration from the TFTP servers. Whenever any IoT device connected to blockchain system requests for updating the configuration or settings, the TFTP server downloads the information and checks for the authentication of the user. After user identification the downloading and updating of the new setup takes place. The proposed architecture is now taking on an average 0.8 seconds to download the configuration from the device and update the same. The blockchain is thus successfully identifying the device and monitoring the IoT devices connected to it. The framework is also updating the requests made from the devices security without tampering the privacy of the information which is beneficial for both utilities and customers.

\section{Conclusion and future work}

This paper proposed asoftware architecture for smart meters using integrated IoT and blockchain technology. The information regarding the consumption of electricity and gas were monitored and a communication protocol is implemented to provide authentication and access to the user through the blockchain framework. After user validation the information will be provided to the utilities which can manage the TOU and supply-demand trade off. The proposed architecture is successfully updating the configuration in the middleware but the time taken is high by few milliseconds than the other servers like SSH and FTP. Thus, our future work will concentrate mainly on reducing the time taken to update the configuration. Other vital information from the devices like the battery life, disk space, RAM size and external hardware connection can be included in improved architecture design.

\section{References}

[1] "Grid 2030, a national vision for electricity's second 100 years," United States Department of Energy Office of Electric Transmission and Distribution, Tech. Rep., 2013. [Online]. Available: http: //www.oe.energy.gov/smartgrid.html

[2] H. Akhavan-Hejazi and H. Mohsenian-Rad, "Power systems big data analytics: An assessment of paradigm shift barriers and prospects," vol. 4, pp. 91-100, 112018.

[3] R.R. Mohassel, A. Fung, F. Mohammadi, K. Raahemifar, "A survey on Advanced Metering Infrastructure", Electrical Power and Energy Systems, Vol. 3, Pp. 473-484, 2014

[4] A. Elmachtoub, V. mname Gupta, and M. mname Hamilton, "The value of personalized pricing," SSRN Electronic Journal, 012018.

[5] Ofgem, "Making a positive difference for energy consumers," [Online]. Available: https://www.ofgem.gov.uk/

[6] IEA, "Power marketers are increasing their share of U.S. retail electricity sales," [Online]. Available: https://www.eia.gov/todayinenergy/detail.php?id=36415 
Journal of Ubiquitous Computing and Communication Technologies (UCCT) (2020)

Vol.02/ No. 01 Pages: 48-58

https://www.irojournals.com/jucct/

DOI: https://doi.org/10.36548/jucct.2020.1.006

[7] S. Sendra, J. Lloret, M. García, J. F. Toledo, Power saving and energy optimization techniques for wireless sensor networks, Journal of communications, 6 (6), 439-459. 2011

[8] A. Khameis, S. Rashed, A. Abou-Elnour, M. Tarique, ZigBee Based Optimal Scheduling System for Home Appliances in the United Arab Emirates, Network Protocols and Algorithms, Vol 7, No 2 (2015).Pp. 60-80

[9] Open Meter Project, available at: http://www.dlms.com/downloads/openmeterdlmsuadissemination090130.pdf [Last access June 15, 2016]

[10] S. Nakamoto. Bitcoin: A peer-to-peer electronic cash system, 2008. Available at https://bitcoin. org/bitcoin.pdf.

[11] E. Monsing, J. Mather, and S. Moura. Blockchains for decentralized optimization of energy resources in microgrid networks. In Conference on Control Technology and Applications (CCTA),pages2164-2171.IEEE, 2017.

[12] Yakubov,A.;Shbair,W.M.;Wallbom,A.;Sanda,D.;State,R.AblockchainbasedPKImanagementframework. In Proceedings of the NOMS 2018-2018 IEEE/IFIP Network Operations and Management Symposium, Taipei, Taiwan, 23-27 April 2018; IEEE: Piscataway, NJ, USA, 2018; pp. 1-6.

[13] https://data.london.gov.uk/dataset/smartmeter-energy-use-data-in-london-households

[14] https://data.london.gov.uk/dataset/smartmeter-energy-use-data-in-london-households

[15] https://www.researchgate.net/post/Can anybody provide annotated dataset of residential_s mart_meter_data_at_different_granularity_levels

\section{Authors Biography}

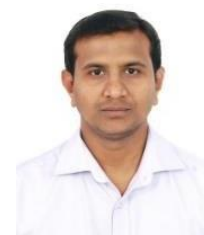

Thilak Raj Lakshmaiah was born in Bangalore, Karnataka, India. He received Bachelor of Engineering in Computer Science from VisveswarayaTechnological University in 2006 and Post Graduate Diploma in Management from Indian Institute of Management, Calcutta, India.Back in 2006, he started the carrier as Data base administrator and thereafter worked in the capacity of ETL designer, Technical Lead, Technical Architect and now as Data Architect. Over the years, hehas been consulting multiple global clients in their Digital Transformation, Data Warehouse and Data Strategy, BI reporting and Analytics and Modernization journey of their legacy applications.Currently he is working as Manager expert in CGI Information Systems and Management Consultants Private Ltd. 
Journal of Ubiquitous Computing and Communication Technologies (UCCT) (2020)

Vol.02/ No. 01 Pages: 48-58

https://www.irojournals.com/jucct/

DOI: https://doi.org/10.36548/jucct.2020.1.006

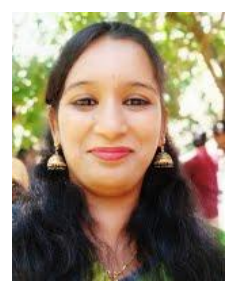

Dr.MadhuraShivaraju was born in Bangalore, Karnataka, India. She received PhD in video processing from VisveswarayaTechnological University in 2018. MTech degree fromMSRIT, Bangalore in 2011. She is currently working as Assistant Professor in Department of Electronics and Communication in RV Institute of Technology and Management, Bangalore. She teaching experience of 9 years with research experience of 7 years. Her research area includes Digital Signal Processing and Video/ Image processing. 